\title{
A Framework for Dynamic Power Management at Network Level
}

\author{
Kamlesh Kumar Verma \\ Department of Computer Science \\ Babasaheb Bhimrao Ambedkar University \\ Vidya Vihar Raebareily Road \\ Lucknow (UP) 226025, INDIA
}

\author{
Vipin Saxena \\ Department of Computer Science \\ Babasaheb Bhimrao Ambedkar University \\ Vidya Vihar Raebareily Road \\ Lucknow (UP) 226025, INDIA
}

\begin{abstract}
In the current scenario of computing, distributing is gaining popularity as many of users are connected with their handheld device across the globe. The connected device generally emits the energy which is of different categories and it is major challenge to optimize the entire energy of the system. The present paper proposes a frame work for the dynamic power management for completion of tasks in minimum time frame which are routed from one node to another node across the network. For this purpose, a step topological network is selected at the application level of the model. Computed results are depicted for the dynamic power management in the forms of graphs.
\end{abstract}

\section{Keywords}

Step Network System, Energy Consumption, Dynamic Power Management, Application Level, System Level, Hardware Component Level. Distributed Computing.

\section{INTRODUCTION}

In the recent years, energy efficient computing is a major challenging area of research. It is mainly depending on hardware or software computing components. The hardware components are as processors, storages, and picture display devices. The performance and power management of the distributed network system are very effective. It is depending on the contributions of networks nodes. In distributed computing systems, the data stores on networks, the nodes must be switched on and connected to each computer. If the participating nodes are wireless then it is more challenging. On network level, the dynamic power management classifies into two types of components i.e. sleep modes or slow down and idle modes. It is a way of reducing power consumption. The dynamic power management has been proposed by the heuristic method for stochastic optimization technique. The heuristic dynamic power management technique is used for time out transmission. The stochastic theory is based on stochastic model of service request. The service requests are of three types. Firstly, each time, a new job process is generated and then searches for procedure to find the minimum power execution sequence, the sequence has also be repeated. Secondly, this strategy does not effective of reducing the system energy by changing the working stage of devices with multi level stage. Thirdly, the exact knowledge of the device uses a process before the job is scheduled. A power management has attached to device components, system architectures, system resources that are shared among these device components. The application level scheduling is requiring the power management for system. The numbers of power saving techniques have been related into many types of standards and protocol eg. Power management in network topology and the power mode in IEEE 802.3 wire network.
The network topology is on network break down. Then a local area network (LAN) card is working in power saving mode and depends on data flow. The dynamic power management is depending on software also.

This paper proposes for network level dynamic power management architecture. It is decomposed into the system level, application level, and component level. This division is integrating of various power management techniques. Application level supports the administrative level handling which is contributing to the users for share system level, and the flow control depends on part level. This paper presents the design, implementation, and network evaluation, wire network device architecture that enables full device functionality, availability, and extended device life. Wire line networks are unique, they have finite times. In large scale distributed network devices such as desktop, the power management is used to extend device.

\section{RELATED WORK}

Let us briefly explain some of the important references related to the work. Hwang and $\mathrm{Wu}[1]$ have described a system level power management technique for power saving. There was a new method for system shutdown developed by authors. Authors had introduced two mechanisms, (i) prediction-miss correction (ii) pre-wakeup. Benini et al. [2] have explained the dynamic power management technique for reducing the power consumption of complex network systems. It is performance and power consumption depended on workload. Qiu and Pedram [3] have invented a theory for Stochastic Modelling of a Power-Management System. Rodoplu and Volkan [4] have discovered a distributed theory for positionbased network protocol optimization for minimum energy consumption in wireless networks. They have supported end to end communications. Simunic et al. [5] have described the algorithms for reducing energy consumption at the systemlevels in low-power states. There are two classes of defining algorithms and proposed for performance measurement (i) timeout and (ii) predictive. They have categorized the algorithms based on stochastic for Performance Measurement and Time Indexed Semi Markov decision process (TISMDP) model in performance. Irani and Gupta [6] have found the strategies for "online" Dynamic Power Management (DPM). In this paper, they have two contributions (1) a theoretical basis for the analysis of DPM for systems with multiple powers (2) the competitive algorithm based on probabilistically generated inputs that improve the competitive ratio over deterministic strategies. Sorber et al. [7] have described the design, implementation, and evaluation of Hierarchical Power Management Architecture (HPMA) for mobile systems. Fernando et al. [8] described the cost optimization method in digital learning region. The method is based on maximum estimation in heterogeneous networks of 
Information Communication Technology (ICTs) like as resource utilisation for educational environments. Ren and Marculescu [9] have discussed a hierarchical technique for DPM under non stationary service requests. The dynamic power management has targeted at extending battery life by low power modes when there is a reduced demand for service. Static power management strategies can effect to poor performance or unwanted power. Jun et al. [10] has found the Disruption Tolerant Networks (DTNs) technique in mobile computing. The DTN's are useful for wireless mobile networks in end to end communication. They evaluated the energy consumed by wireless communication. The energy can be using the low-power radio and high-power radio. Chelius and Mignon [11] have explained the minimization of the power consumption to the nodes of wireless sensor networks for lower and upper bound broadcasting. The theory is based on mathematical programming problem in sensor networks. Huang et al. [12] have described the topology for optimized link state routing in mobile ad hoc network MANET routing protocols. Cao et al. [13] have discussed, the green computing which explores the potential energy savings for the direct parallel implementation, and this is used with a large-scale computational biology application. There are two power approaches for direct for the CPU speed power saving. The parallel workload of the effective application is needed for power management. Gouvy et al. [14] have invented an energy aware routing algorithm for minimizing the energy consumption through mobile with connectivity in sensor networks. Elavarasan. R et al. [15] have invented an algorithm for delay aware network structure for WSN's with consecutive data collection and reduction of energy consumption without data losses from peer to peer data transmission has been proposed. Limmer et al. [16] have found a numerical method for simulations and optimizations performance in grid computing. Sharma and Nitin [17] have described a simulation method in real time distributed system for finding the entropy. Balachennaiah et al. [18] have described an algorithm based technique for optimization the control of power loss and voltage stability in transmission time. Quwaider et al. [19] have invented the theory for the mobile cloud computing and mobile cloud resources. Lyu et al. [20] have defined high performance scheduling model with a cloud sensor system. In cloud system, scheduling has performed optimization by Zero One programming method by task models. Han et al. [21] have invented a algorithm for distributed hierarchical game based algorithm for power allocation in Orthogonal Frequency Division Multiple Access (OFDMA) networks system. Moety et al. [22] have described the optimization models for power delay minimization theorem in green wireless networks. Huang et al. [23] have invented the cloud data redundancy for high reliable cloud storage systems and also demonstrate the improvement of saving of data redundancy computation in networks system. Hassan, et al. [24] has discovered the evaluation the scalability and performance of High Performance Cloud Computing (HPCC) on Microsoft Azure Cloud Systems (MACS). Agostini et al. [25] have found the management of strategic multi partner SME small and medium enterprises networks introduce the concepts of heavy weight, lightweight and internal or external network. Alonso et al. [26] have described the management for power consumption in fat tree communication networks. It is depended on network bandwidth in transport requirement. Zdraveski et al. [27] have described an algorithm for the load balancing in power distribution networks in cloud system. Walkowiak et al. [28] have discussed two methods Popular Wavelength Division Multiplexing (WDM) and a new emerging approach of Elastic Optical Network (EON). Ayyoub et al. [29] have invented the theory for the optimizing techniques for ultra scale cloud computing data centres. Jiang et al. [30] have discussed a probabilistic challenge for the users files which are available and stored specified cloud server. the cloud infrastructure with some reasonable as (i) Rational Economic Security Model, (ii) Semi Security Model of cloud servers. Mershad et al. [31] have given a mathematical model for evaluation and the utilization of a big data in cloud data centre. Nabiel et al [32] have described the cost optimization approaches for scientific workflow in cloud and grid computing. $\mathrm{He}$ and $\mathrm{Li}$ [33] described a fast diagnosis algorithm for communication network in high performance computers. Banditwattanawong et al. [34] have invented i-cloud (intelligent cloud) performances which were stable and close to those of infinite cache size. Damian et al. [35] have given a theory (i) quality of service (ii) a general control theoretic approach for cloud service (iii) the proposed language and control.

\section{PROPOSED MODEL}

Network system level dynamic power management architecture is represented in the following figure. The model is divided into three parts which are described in Figure 1.

\subsection{Application Level}

In the application level, many users work with server system. The server is working as distributed computing system as a data core network. All others send the application for processing with server interaction with the network. The server permits to all users for synchronize process. The synchronized process is controlled by the administrator such types of user operation by sequence. The users are interconnected across the step network as shown below:

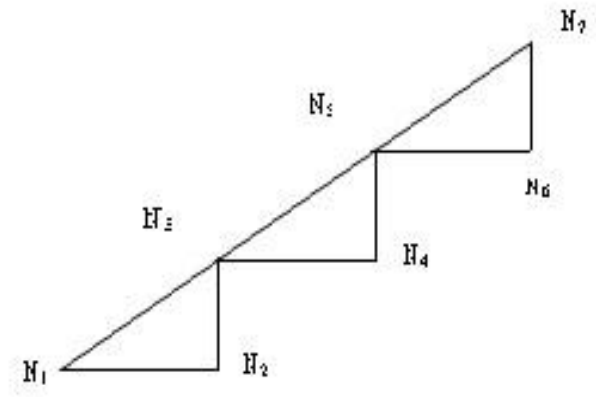

Figure2. A Step Network System

\subsection{System Level}

At the system level, the application server works on system level component. There are many services which request to organize by the computing as first come first serve. Afterthat, the service request is becoming complete then the next step is going to service flow control. The Service Flow Control also works with service request in minimum duration of time interval with related to request. It is working with data process and depends on throughput process and latency. The latency must be minimized in duration of such process operations. 


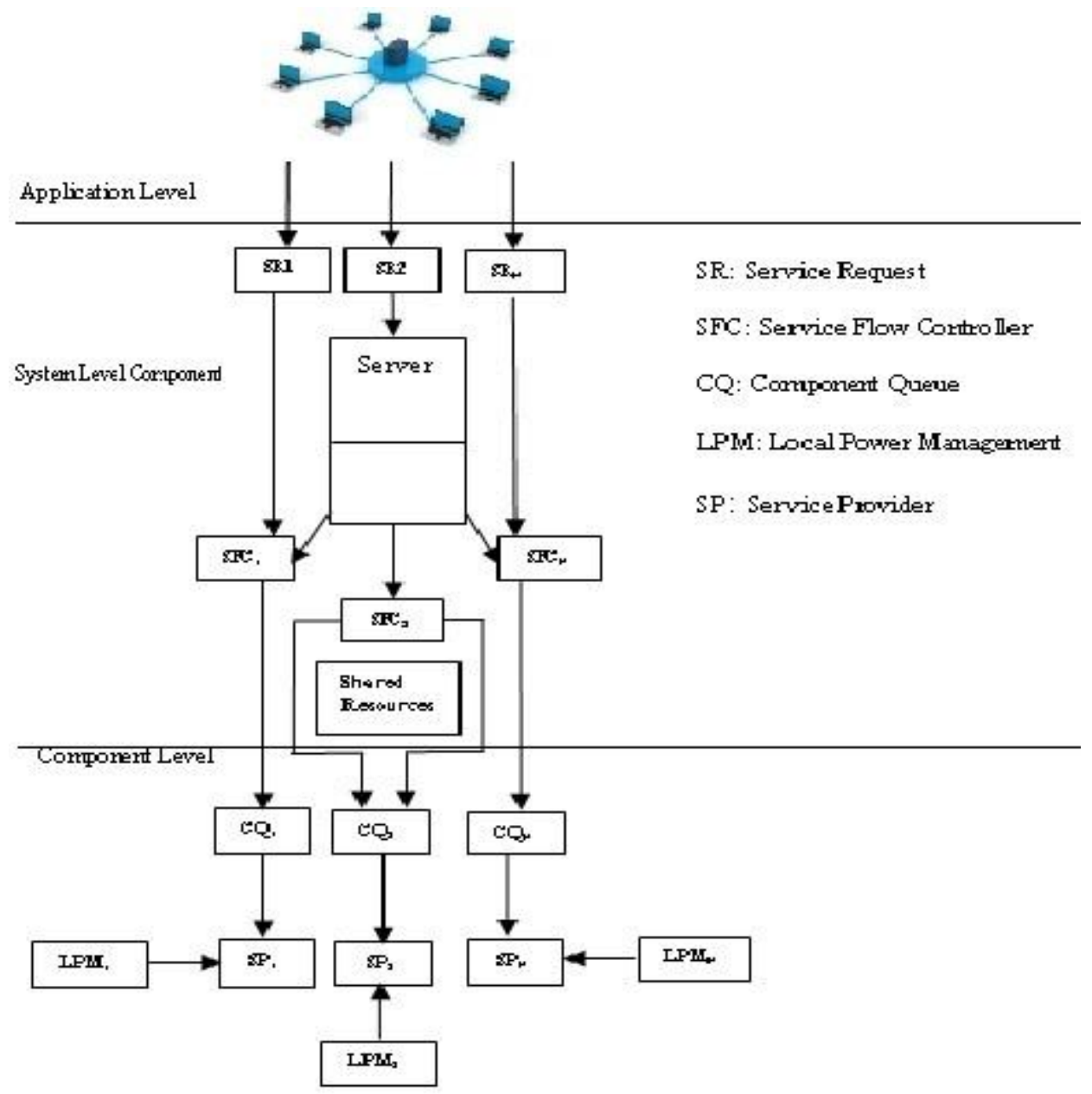

Figure 1. A Network System Level Dynamic Power Management Architecture

\subsection{Hardware Component Level}

The hardware component level is attached to the computers. The every computer has own power management system known as local power management. Every service provider has own request related to control queue request in the synchronization process. All service providers interact with common resources and control on all components which request as the throughput process. In the above diagram, if the set of states (S) for all servers is $S=\left\{s_{1}, s_{2} \ldots \ldots \ldots \ldots \ldots s_{n}\right\}$ for all possible states with $A=\left\{a_{1}, a_{2} \ldots \ldots \ldots \ldots \ldots a_{n}\right\}$ for all possible actions, then the relation model is given below.

$R: S \times A \rightarrow R$ Specifies for taking action in state $\mathrm{S}$

Now let us define the transition model for the process. The transition model specifies probability of transmission from state $\mathrm{i}$ to state $\mathrm{j}$ on taking action a and the specified matrix

$T_{i j}^{a}$ is given below:

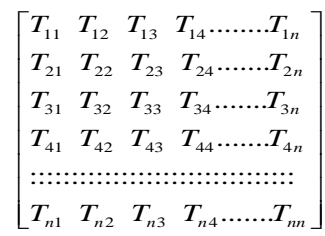

Figure 3. Transition Matrix
The above matrix indicates the process states $\mathrm{T}_{11}, \mathrm{~T}_{22}, \mathrm{~T}_{33}$ $\mathrm{T}_{44 \ldots \ldots .} \mathrm{T}_{\mathrm{nn}}$, is diagonal which shows the process is ideal for next state and data throughput. If the transmission state $i$ to $j$ on taking action a and defines the specified matrix $\mathrm{T}_{\mathrm{ij}}{ }^{\mathrm{a}}=$ $\mathrm{T}_{11}, \mathrm{~T}_{22}, \mathrm{~T}_{33}, \mathrm{~T}_{44} \ldots \ldots \mathrm{T}_{\mathrm{nn}}$ is ideal. In the transmission matrix $\mathrm{T}_{11}, \mathrm{~T}_{22}, \mathrm{~T}_{33}, \mathrm{~T}_{44} \ldots \ldots \mathrm{T}_{\mathrm{nn}}$ are ideal, and all remaining may be in the waiting state for next state or failed transmission.

\section{NUMERICAL RESULTS AND DISCUSSIONS}

One can compute extensive numerical values as the size of step network grows. Authors have computed for five nodes as shown in the figure 2 by considering the file size of $0.6 \mathrm{MB}$ which is to be transmitted across the network. The transition time is computed for all possible combinations from $n_{1}$ node to $\mathrm{n}_{5}$ node by using the following:

$$
T_{i j}^{a}=k(\text { Arrival Time }+ \text { Generation Time })
$$

Where $T_{i j}^{a}$ is the transmission time in state action a and $\mathrm{k}$ is the Load Factor. The minimum transition time is also computed by the use of Hungarian Method [35] and it is computed as 3.014 milliseconds when the file is to be routed from $\mathrm{T}_{14}, \mathrm{~T}_{22}, \mathrm{~T}_{31}, \mathrm{~T}_{33}$ and $\mathrm{T}_{55}$. On the basis of above, the transition table is given below: 


$$
\left[\begin{array}{lllll}
0.540 & 0.594 & 0.570 & 0.564 & 0.710 \\
0.560 & 0.588 & 0.564 & 0.688 & 0.656 \\
0.510 & 0.582 & 0.540 & 0.578 & 0.662 \\
0.630 & 0.606 & 0.570 & 0.698 & 0.652 \\
0.688 & 0.735 & 0.757 & 0.751 & 0.782
\end{array}\right]
$$

\section{Figure 4. Transition Table}

The transmission in between of the network is considered as a minimum time for example if two routes exist between $\mathrm{n} 1 \rightarrow \mathrm{n} 4$ i.e. $\mathrm{n} 1 \rightarrow \mathrm{n} 2 \rightarrow \mathrm{n} 4$ or $\mathrm{n} 1 \rightarrow \mathrm{n} 3 \rightarrow \mathrm{n} 4$ then that route is selected which consumes minimum transmission time. In such a manner, the data can be transmitted across the step network. Now the DPM is constructed to the point of view of whole System Power Management (SPM). This is utilized to derive a system level policy and component level policy. The above model contains Application Level (AL), Service Queue (SQ), Service Control Flow (SCF) and Analysis Service Provider (ASP). The working functionality of entire system is shown below:

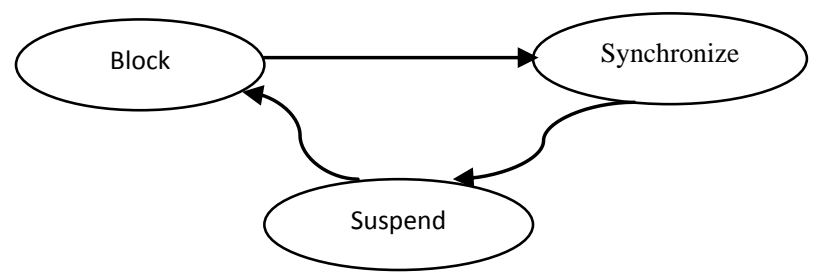

Figure 5. Functions during DPM

The SFC is modeled as a stationary. The state set of the SFC as SSFC $=\{$ Block, Suspend, Synchronize $\}$ and action flow of SFC is the set of action, ASFC $=$ \{Goto_Synchronize, Goto_Suspend, Goto_Block\}.The details of state and transition of the SFC are explained.

(a) Synchronize: In this state, the SFC generates a run state process and classified two types of synchronized process in whole Universal Power Management (UPM) (i) Synchronized Service Queue (SSQ) (ii) None Synchronized Service Queue (NSSQ). The SSQ requests to the Service Provider (SP) on response time for next process zone. SP is decided in the process of that of fixed time. If fixed time for process is not completed, then Goto NSSQ and vice versa.

(b) Suspend: In this state, the SFC continuously moves from SSQ to the SP. The SP will take up and provide requested services. Now a new component is added in that time Component Queue (CQ). The CQ is available on component level policy. The process is in SSQ by the acknowledged from $\mathrm{SP}$ at service request on the queue (SRQ). The SRQ moves towards a head for throughput one by one and burst it.

(c) Block: In this state the SFC blocks all incoming SRQ from the entering the CQ of the SP. The main objective of the blocking various non synchronized processes is reduced and wake up times of SP and extend time utility the SP, synchronized process during sleep mode. Therefore,

The operation of request for service $(\mathrm{SR})=$ Waiting Time + Service Operations.

It is clear from the above that non synchronization process over the network gives increase in the transmission process with heavy load on system. The packet delivery fraction in service flow provider is based on the ratio of data packets transmission to those generated by sources. During the routing, load depends on routing packets sent per transmitted packets at the destination. Let us consider the SP with the fixed time out policy and hard disk drive consists of two power states. (i.e. Active $=2.1$ watt, Low power $=0.65$ watt) and the transition power and time between the two states are 1.4 milliseconds and 0.4 milliseconds; the local power manager has two states as shown below in figure 4 .

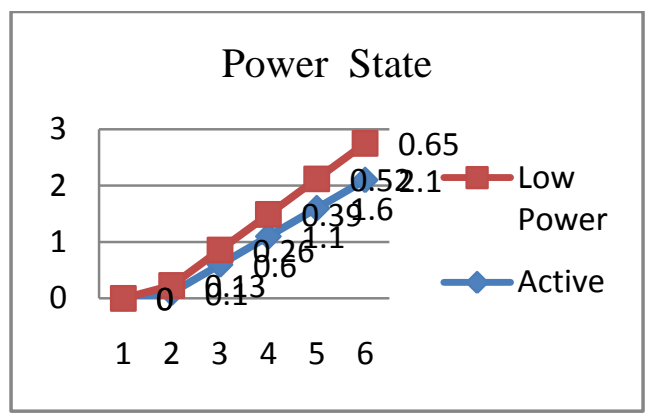

Figure 6. Power State Process from User to Server

The transmission processes from user have local power management and the initial time depends on the user performance related to time and energy from time interval. If the initial time is 0.7 second and the power consumption is 0.2 watt, then transmission process is shown below:

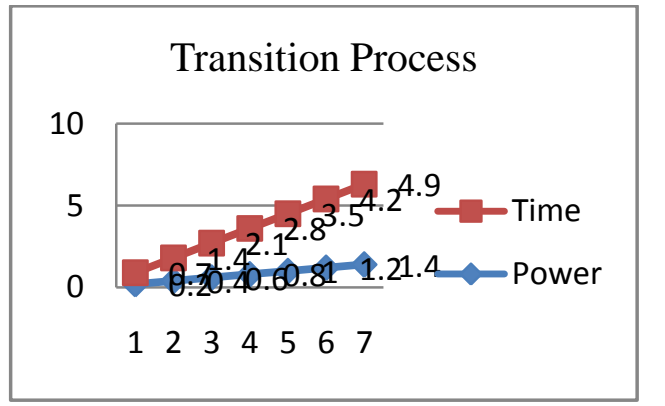

Figure 7. Transmission Process across Step Network

\section{CONCLUDING REMARKS}

In this paper, a model is proposed for distribution of the resources with three different levels across the distributed networks in which users are connected across the newly developed step network. The transmission time is optimized by the Hungarian technique by considering the limited nodes but it can be extended up to the finite numbers of nodes connected across the step topological network. In the proposed approach, the model is decomposed into three parts namely application level system, component level and system level. Dynamic power management technique is used for optimization of the transmission of the processes. In above table has taken as per each system. The experimental results has evaluated on five system connected over network server.

\section{REFERENCES}

[1] Hwang, C-H. and Wu, A. "A Predictive System Shutdown Method for Energy Saving of Event Driven Computation". Proceedings International Conference on Computer Aided Design, San Jose, California, pp. 28 32, IEEE Xplore Digital Library 1997.

[2] Benini, L., Paleologo, G., Bogliolo, A., De Micheli G., "Policy Optimization for Dynamic Power Management". IEEE Trans Comput Aided Des pp. 813-833, 1999.

[3] Qiu Q, Wu., and Q, Pedram M., "Stochastic Modelling of a Power-Managed System Construction and 
Optimization”. IEEE Trans Comput Aided Des pp 12001217, 2001.

[4] Simunic, T., Benini, L., Glynn, P., De, Micheli G. "Event-Driven Power Management". IEEE Trans Comput Aided Des, pp 840-857, 2001.

[5] Bhat, UN. "Elements of Applied Stochastic Processes". Wiley-Inter Science, 2002.

[6] Irani, S., Shukla, S., Gupta, R.," Competitive Analysis of Dynamic Power Management Strategies for Systems with Multiple Powers Saving States". Proceedings Design and Test in Europe, pp. 117-123, 2002.

[7] Sorber, J., Banerjee, N., Corner, M., Rollins S, Turducken., "Hierarchical Power Management for Mobile Devices". Proc Mobi Systems, Jun Seattle (WA). pp. 261-274, 2002.

[8] Ren, Z., Krogh, BH., Marculescu, R., "Hierarchical Adaptive Dynamic Power Management". IEEE Trans. Comput. , pp 409-420, 2005.

[9] Theocharous, G., Mannor, S, Shah., N, Gandhi., P, Kveton., B, Siddiqi., Yu, CH.,"Machine Learning for A adaptive Power Management". Intel Technol J, pp 299-312, 2006.

[10] Jun, H., Ammar, M., Corner, M., Zegura, E., "Hierarchical power management in disruption Tolerant Networks with Traffic-Aware Optimization". Proceedings SIGCOMM Workshop on Chants, Paris, France, pp. 245-252, 2006.

[11] Chelius, Guillaume., Fleury, Eric., Mignon,Thierry., "Lower and Upper Bounds for Minimum Energy Broadcast and Sensing Problems in Sensor Networks". The International Journal of Parallel, Emergent and Distributed Systems, Vol. 21, No. 6, pp 405-422. www.tandfonline.com, December 2006.

[12] Huang, Yangcheng., Bhatti, Saleem., Sørensen, SørenAksel., "The Impact of Topology Update Strategies on the Performance of a Proactive MANET Routing Protocol". Int. J. of Parallel, Emergent and Distributed Systems Vol. 23, No. 6, pp 47-460, http://www.tandfonline.com, December 2008.

[13] Cao, Zhenwei., Easterling, David., "Power Saving Experiments for Large-Scale Global Optimisation". International Journal of Parallel, Emergent and Distributed Systems Vol. 25, No. 5, pp 381-400, http://www.tandfonline.com, October 2010.

[14] Gouvy, Nicolas., Hamouda, Essia., Mitton, Nathalie., Simplot-Ryl, David., "Minimising Energy Consumption through Mobility with Connectivity Preservation in Sensor Networks". International Journal of Parallel, Emergent and Distributed Systems Vol. 27, No. 6, pp 521-540, www.tandfonline.com, December 2012.

[15] Elavarasan, R., and Steffi, Perpectual A., "Energy Efficient Data Collections Structure for Wireless Sensor Networks". International Journal of Science and Research (IJSR), ISSN 2319--7064, Volume 3 Issue 4, April 2014

[16] Bruschi, Roberto., Lago, Paolo., Lombardo, Alfio., Schembra, Giovanni., "Modeling Power Management in Networked Devices". Computer Communications. pp 95-109, www.elsevier.com/locate/com, 2014.

[17] Sharma, Rashmi., and Nitin. "Entropy, a New Dynamics Governing Parameter in Real Time Distributed System: A Simulation Study”. Int. J. of Parallel, Emergent and Distributed Systems,Vol. 29, No. 6, pp 562-586, www.tandfonline.com/loi/gpaa20,2014.

[18] Balachennaiah, P., Suryakalavathi, M., Nagendra, Palukuru., "Optimizing Real Power Loss and Voltage Stability Limit of a Large Transmission Network using Firefly Algorithm". Engineering Science and Technology, ttp://www.elsevier.com/locate/jestch,2015.

[19] Quwaider, Muhanna d., Al-Alyyoub, Mahmoud., Jararweh, Yaser., Duwairi, Rehab., "Experimental Framework for Mobile Cloud Computing System". Procedia Computer Science 52, pp 1147 - 1152, www.sciencedirect.com,2015.

[20] Lyu, Yongqiang., Yan, Fanxi., Chen, Yu., Wang,Dan., Agoulmine,Nazim.,"High-Performance Scheduling Model for Multisensor Gateway of Cloud Sensor SystemBased Smart- Living”. Information Fusion,pp 42-56, www.elsevier.com/locate/inffus, 2015.

[21] Hn, Song. Li., Xin, bin., Liu, Zhixin., Guan, Xinping., "Distributed Hierarchical Game-Based Algorithm for Downlink Power Allocation in OFDMA Femto Cell Networks".Computer Networks, pp 1-13, www.elsevier.com/locate/comnet,2015.

[22] Moety,Farah., Lahoud, Samer., Cousin,Bernard., Khawam,Kinda., "Optimization Models for the Joint Power - Delay Minimization Problem in Green Wireless Access Networks."

[23] Huang, Zh en., Chen, Jinbang., Lin, Yisong., You, Pengfei., Peng, Yuxing.,"Minimizing Data Redundancy for High Reliable Cloud Storage Systems". Computer Networks pp 164-177, www.elsevier.com/locate/ comnet, 2015 .

[24] A., Hassan., Hanan, A., Mohamed, Shimaa., M Sheta, Walaa., "Scalability and Communication Performance of HPC on Azure Cloud". Egyptian Informatics Journal, www.sciencedirect.com, 2015.

[25] Agostini n, Lara. Filippini, Roberto. Nosella, Anna., "Management and Performance of Strategic Multipartner SME networks". Int. J. Production Economics pp 376-390, www.elsevier.com/locate/ijpe, 2015.

[26] Alonso, M., Coll, S., Martínez , J.M ., Santonja, V., P, López., " Power Consumption Management in Fat-Tree inter Connection Networks". Parallel Computing pp 5980, www.elsevier.com/locate/parco,2015.

[27] Zdraveski, Vladimir., Todorovski, Mirko., Kocarev, Ljupco., "Dynamic Intelligent Load Balancing in Power Distribution Networks". Electrical Power and Energy Systems pp 157-162, www.elsevier.com/ locate/ijepes, 2015.

[28] Krzysztof, Walkowiak., Michał, Woźniak., Mirosław, Klinkowski., Wojciech, Kmiecik., "Optical Networks for Cost-Efficient and Scalable Provisioning of Big Data Traffic”. Inter J. of Parallel, Emergent and Distributed 
Systems, Vol. 30, No. 1, pp 15-28, www.tandfonline.com/action/journalInformation , 2015.

[29] Al-Ayyoub, Mahmoud., et al. "Optimizing Expansion Strategies for Ultrascale Cloud Computing Data Centers." Simulation Modelling Practice and Theory pp 15-29, www.elsevier.com/locate/simpat,2015.

[30] Jiang, Tao., et al. "Towards Secure and Reliable Cloud Storage Against Data Re-Outsourcing".Future Generation Computer Systems, pp 86-94, www.elsevier.com/ locate/fgcs, 2015.

[31] Mershad N, Khaleel., Artail, Hassan., Saghir, Mazen., Hajj, Hazem., Awad, Mariette., "A Mathematical Model to Analyze the Utilization of a Cloud Data Center Middleware". Journal of Network and Computer Applications, pp 399-415,www.elsevier.com/locate/ jnca, 2016

[32] Alkhanak, EhabNabie., Peck Lee,Sai., Rezaei, Reza., Parizi, Reza Meimandi., "Cost Optimization
Approaches for Scientific Work Flow Scheduling in Cloud and Grid Computing :A Review, Classifications and Open Issues". The Journal of Sys. and Software, pp 1-26, www.elsevier.com/locate/jss,2016.

[33] He, Li., and Li, Furong., “A Fast Diagnosis Algorithm for Interconnection Network in High Performance Computers".'International Journal of Parallel, Emergent and Distributed Systems, Vol.31, No.1, pp 3446, www.tandfonline.com/action/journalInformation, 2016.

[34] Banditwattanawong, Thepparit., Masdisornchote, Masawee., Thayopas, Putchong.,"Multi-Provider Cloud Computing Network Infrastructure Optimization".Future Generation Computer Systems, pp 116-128, www.elsevier.com/locate/fgcs,2016.

[35] Serrano, Damián., et al. "SLA Guarantees for Cloud Services". Future Generation Computer Systems, pp 233-246, www.elsevier.com/locate/fgcs,2016. 